\title{
Efficiency Improvement of a High Dynamic BLDC Linear Motor by Multiphase Control
}

\author{
Joris Lemmens, Kristof Mulier and Johan Driesen \\ Dept. of Electrical Engineering ESAT-ELECTA \\ Katholieke Universiteit Leuven \\ B-3001 Leuven, Belgium
}

\author{
Kris Vanvlasselaer, Stijn Goossens and Wim Symens \\ Flanders' Mechatronics Technology Centre (FMTC) \\ B-3001 Leuven, Belgium
}

\begin{abstract}
This paper proposes a multiphase control strategy for a high dynamic brushless DC linear motor as an alternative for conventional three-phase field-oriented control. Analysis of the magnetic field waveforms shows that three-phase control is not optimal for the 6-slot 7-pole motor topology. Therefore, a multiphase control strategy is elaborated which injects currents proportional to the electromotive force into each of the nine stator coil groups. This results in a maximal alignment force per ampere between the stator and slider fields. Furthermore, only the stator coils with a useful contribution to the slider force are excitated. According to the simulation model, this yields a significant reduction of joule and iron losses for equal dynamic performance. The new strategy is implemented on a dSPACE rapid prototyping platform, controlling a custom-built multiphase converter. Measurements confirm the simulation results and show a significant reduction of power consumption compared to conventional three-phase control
\end{abstract}

\section{INTRODUCTION}

In high-tech manufacturing machines, linear motions are usually performed by a rotating motor in combination with a mechanical transformation element, for example a cam follower mechanism. This is reliable and proven technology, but directly using an electromagnetic linear actuator instead has potential benefits in terms of motion trajectory flexibility, compactness and maintenance-free operation. The challenge however is the need for very large accelerations in modern manufacturing machines. Previous work [1] addressed this issue and presented a high dynamic BLDC linear motor prototype as shown in Fig. 1. The motor was controlled with an off-the-shelf three-phase drive and performed well in terms of dynamics with accelerations up to $2000 \mathrm{~m} / \mathrm{s}^{2}$. However, in terms of energy efficiency, a large potential for improvement still remained. In this view, alternative stator winding configurations and control strategies should be considered. In the conventional three-phase configuration, all coils are excitated when driving the actuator. As can be seen in Fig. 1, the slider length is several times shorter than the maximum stroke length. Hence, a possible way to reduce energy consumption is to switch off coils which are not contributing to the force generation. This is a common approach for active way linear motors with a long stator consisting of a number of independently supplied sectors [2]-[5]. A similar approach does not use the concept of sectors, but supplies each coil (group) separately with a multiphase converter [6], [7]. Because only the useful coils are excitated, losses can be reduced significantly. A drawback of this approach is that the increased number of phases requires additional power electronic switching devices, adding to the cost and complexity of the system.

The goal of this paper is to assess the added value of a multiphase control strategy with separate coil supply for the linear motor prototype. The paper start with an overview of the BLDC linear motor topology with a basic analysis of the magnetic field interactions and force equations. This will elucidate why the conventional three-phase configuration is not efficient regarding force generation. A more detailed analysis of the magnetic field waveforms is available in [8]. From the analysis, an improved multiphase control strategy is proposed. Next, a Simulink Model estimates the performance gain of the new control strategy. Finally, power consumption measurements on an experimental setup, including a custombuilt multiphase converter, are presented.

\section{High DyNAMIC BLDC LinEAR MOTOR}

Fig. 1 shows the fractional-slot BLDC linear motor design. The stator consists of a double-sided iron core with 18 teeth, each containing a concentrated winding. The moving part of the actuator (slider) includes seven $\mathrm{NdFeB}$ magnets embedded in a lightweight composite material. In order to reduce cogging force, the permanent magnets are placed in a slanted position. The slider is supported by dry plain bearings on guiding rails. Total stator core length is $360 \mathrm{~mm}$ with a maximum stroke length of $250 \mathrm{~mm}$. The cross-section portrayed in Fig. 2 gives a good view of the motor topology. Seven slider magnets are covered by six stator teeth (6-slot, 7-pole ratio) and every coil unwraps two neighboring teeth, resulting in 9 coils in total. In the conventional three-phase configuration, the coils are grouped in a star-connection in order to control the motor with an off-the-shelf three-phase motor drive. Each phase contains three series-connected coils resulting in the following coil groups: 1-4-7, 2-5-8 and 3-6-9.

\section{A. Stator and Slider Field Interaction}

Consider a 6-slot 7-pole BLDC linear motor with an infinitely long slider moving at a constant velocity. The magnetic field B (flux density) in the air-gap due to the permanent magnets on the slider is shown in Fig. 3 and can be approximated as a standing wave which can be broken down into right and 


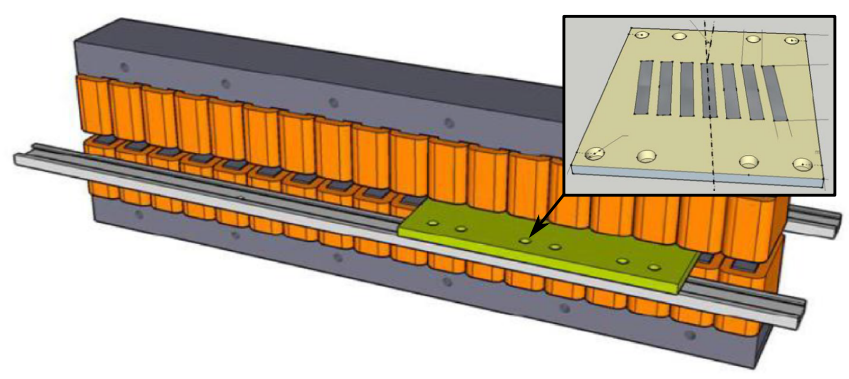

Fig. 1. High dynamic 6-slot 7-pole BLDC linear motor design from [1].

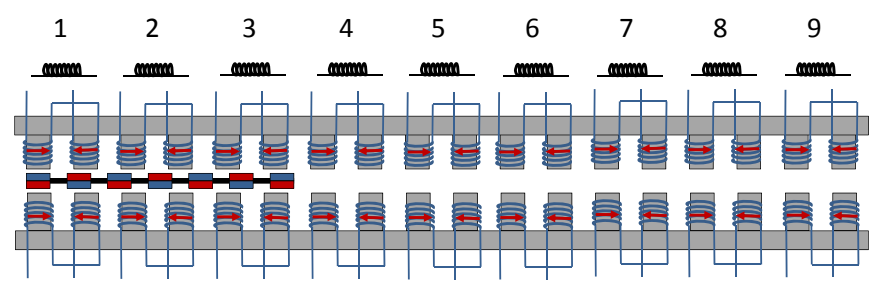

Fig. 2. Cross-section of the linear motor showing 9 coil groups.

left traveling sinusoidal harmonic waves. To take the finite length of the slider into account, the harmonics are multiplied with a block-shaped running wave $\delta(\alpha, t)$ with a unity amplitude as shown in Fig. 4. Hence, the permanent magnet field is only present at the slider position. The fundamental right traveling slider field is shown in green in Fig. 5.

When currents are injected into the stator coils, a stator magnetic field is created. In analogy with the slider field, the standing wave can be broken down into left- and right traveling waves. As a result, a force on the slider is generated by the interaction (alignment force) between the harmonic waves of the slider and the stator. Fig. 5 shows the fundamental right traveling magnetic waves (for a finite length slider) in the air gap when a conventional three-phase drive supplies the starconnected coils with three 120 degree phase-shifted currents. The figure illustrates the inefficiency; the optimal 90 degree phase-shift (load angle for maximum alignment force) between stator and slider field is only achieved in the middle of the slider. The phase-shift is higher and lower at the left and right side respectively. Furthermore, the stator field outside

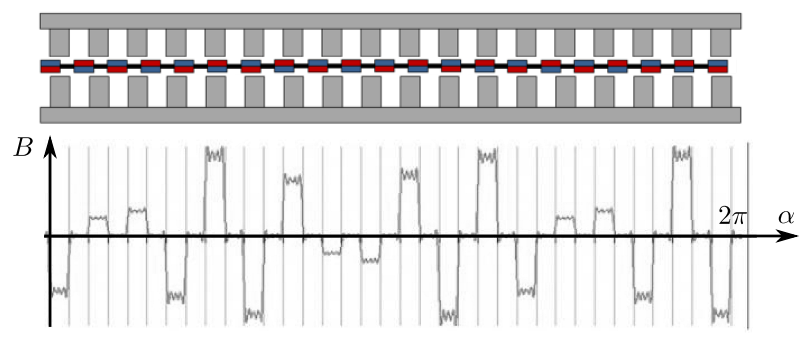

Fig. 3. Permanent magnet field in the air-gap for an infinitely long slider. The resulting standing wave can be broken down into left and right traveling sinusoidal harmonic waves.

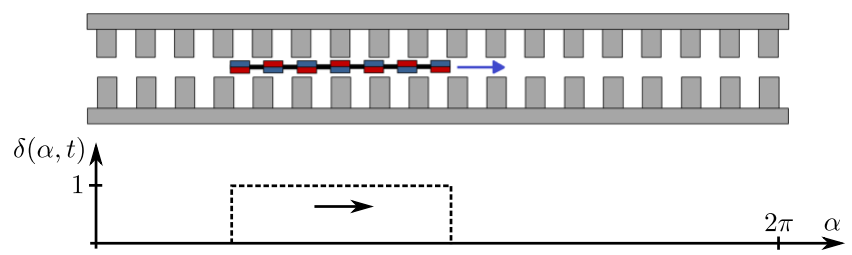

Fig. 4. Running wave $\delta(\alpha, t)$ with unity amplitude moving at slider velocity. The slider field harmonics are multiplied with $\delta(\alpha, t)$ to take the finite length of the slider into account.

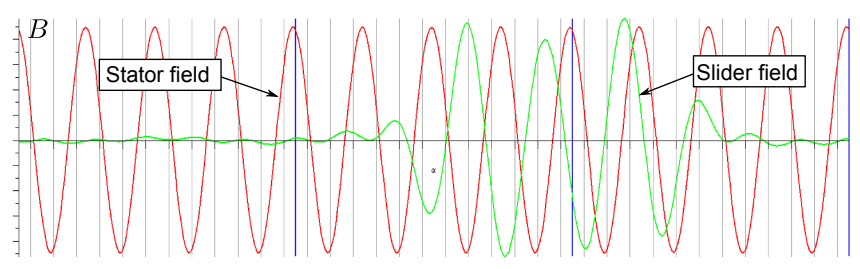

Fig. 5. Fundamental right traveling magnetic field waves of slider and stator with three-phase sinusoidal supply.

the actual slider position has no useful contribution to the total force, it only generates additional losses. Note that the figure only shows the right-running fundamental stator and slider magnetic field waves. Analysis of the interaction between higher harmonics and left-running waves reveals the same inefficiency in force generation [8]. Therefore, the next section will propose an alternative coil connection scheme with a new control strategy for more efficient force generation compared to the conventional three-phase control.

\section{B. Multiphase Control Strategy}

The total electrical force $F$ on the slider can be calculated at each time instant by multiplying the instantaneous normalized electromotive force $E M F_{c, n}$ (at slider velocity $1 \mathrm{~m} / \mathrm{s}$ ) in each coil $\mathrm{c}$ with the coil current $i_{c}$ as given by (1). The first part of this formula represents the alignment force between the fields of stator and slider. The second part represents the parasitic cogging-force. With $\phi_{m}$ the flux of magnet $\mathrm{m}, R_{m}$ the flux path reluctance and $\beta$ the slider position. Because this motor has salient poles, the cogging force is relatively high. This force is partially suppressed by the skewing of the slider magnets and the 6-slot 7-pole topology.

$$
F=\sum_{c} i_{c} \cdot E M F_{c, n}-\sum_{m} \frac{1}{2} \phi_{m}^{2} \frac{\partial R_{m}}{\partial \beta}
$$

The EMF-voltages induced by the moving slider in each of the nine coils, depend of the actual slider position. They have a quasi-sinusoidal shape and are 60 degrees mutually phaseshifted as shown in Fig. 6. With a 2D numerical simulation model of the linear motor magnetic fields, $E M F_{c, n}$ (Fig. 7) in each of the nine coils is calculated and stored in a lookup table for a large number of slider positions.

Equation (1) implies that the most efficient force generation, i.e. the largest alignment force for a given current amplitude, is obtained when the current injected to a coil is in phase 

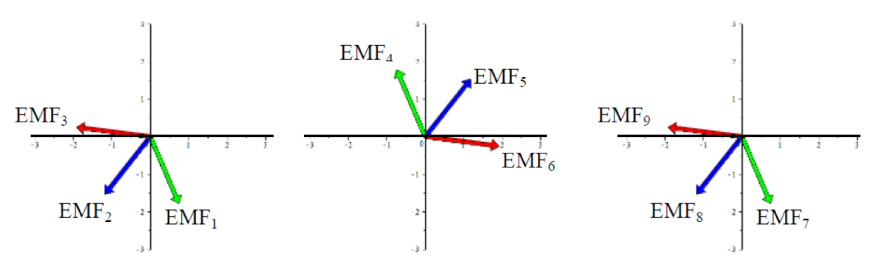

Fig. 6. Phasor representation of the electromotive force in the 9 stator coils
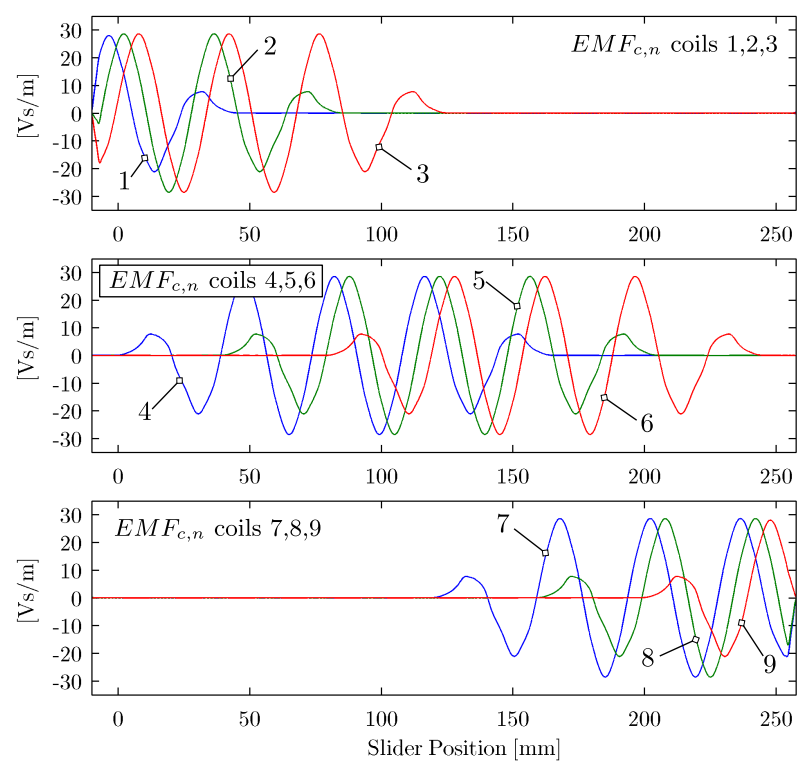

Fig. 7. Normalized EMF (at slider velocity $1 \mathrm{~m} / \mathrm{s}$ ) in each of the 9 stator coils as a function of slider position.

with the EMF. Furthermore, the relative distribution of currents between all coils to generate a certain amount of slider force $F$, can be optimized as well. Assuming a proportionality constant $\kappa$, the optimal current $i_{c}$ through coil $\mathrm{c}$ is:

$$
i_{c}=\kappa \cdot E M F_{c, n}
$$

with $E M F_{c, n}$ the normalized EMF in coil number $\mathrm{c}$ at the actual slider position. The force on the slider due to the current in coil $\mathrm{c}$ is:

$$
F_{c}=i_{c} \cdot E M F_{c, n}=\kappa \cdot E M F_{c, n}^{2}
$$

Considering the force contribution of all stator coils, the total force on the slider is given by:

$$
F=\kappa \cdot \sum_{c} E M F_{c, n}^{2}
$$

Hence, the proportionality constant $\kappa$ can be calculated from the force setpoint $F$ and the normalized EMF in each coil at the actual slider position as shown in Fig. 7:

$$
\kappa=\frac{F}{\sum_{c} E M F_{c, n}^{2}}
$$

Fig. 8 shows the optimal fundamental right traveling stator field in the air gap when each of the nine coils is supplied

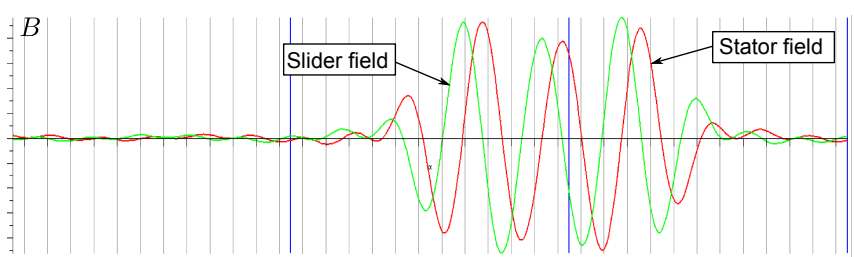

Fig. 8. Fundamental right traveling magnetic field waves of slider and stator Multiphase supply.

with a current $i_{c}$ according to (2). Notice that the stator field is exactly 90 degrees shifted to the right with respect to the slider field (as EMF is the derivative of flux), exerting optimal force on the slider. The load angle between slider and stator fields will be optimal as well for the higher harmonics and left traveling waves which are not shown here. Moreover, the stator field is only present at the slider position, since no current is injected in the non-contributing coils (i.e. coils for which the EMF is nearly zero). As a result, this control strategy yields a more efficient force generation compared to threephase control. It will induce less joule and iron losses when generating a certain amount of force.

A new coil connection scheme and converter topology is required to execute the previously described control strategy. The new multiphase converter should be able to supply each of the nine coils with a current proportional to the EMF. Hence, every coil will be supplied independently with a bidirectional current by means of a nine-phase converter, containing nine H-bridges as shown in Fig. 9.

\section{Simulation}

\section{A. Model Description}

The previously described control strategy was implemented in a Simulink model to verify the dynamic behavior and efficiency improvement potential. Fig. 9 gives a block diagram of the control strategy. It consists of three major parts: a position control loop, a current distribution block and nine current control loops (one for every coil group). The position control loop calculates the necessary force $F_{r e f}$ on the slider to track a desired position profile $x_{r e f}$. This profile can be either a sawtooth or a sinewave with adjustable stroke length and translation frequency. The current distribution block translates the requested force into nine current setpoints $i_{c, r e f}$ according to (2), yielding maximum force generation per ampere. From a lookup table, the current distribution block receives the normalized electromotive force $E M F_{c, n}$ of each coil for the actual slider position $x_{\text {meas }}$. The nine current control loops generate voltage setpoints $U_{c, \text { ref }}$ by means of a PI controller with the voltages $E M F_{c}$ added as a feed-forward term to improve controller's performance. A current limit is set at 10 A. Finally, the voltages are applied to the motor coils by means of a multiphase converter consisting of nine H-bridges which are controlled by sinusoidal pulse-width modulation.

The converter is modelled straightforwardly as a first-order delay. Fig. 10 shows the linear motor model which consist 


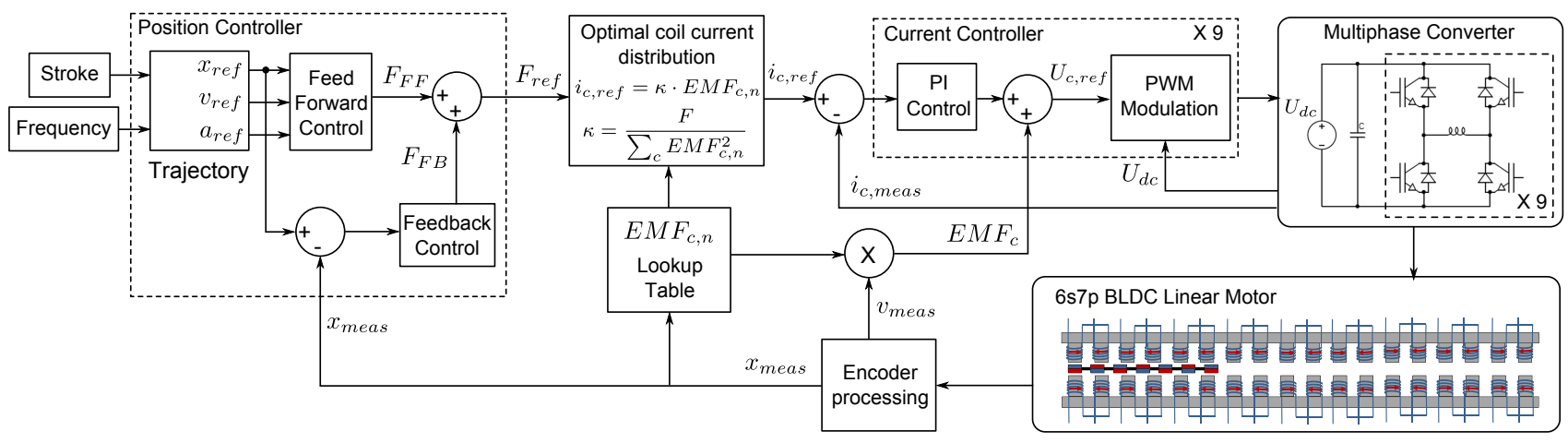

Fig. 9. Overview of the proposed multiphase control strategy.

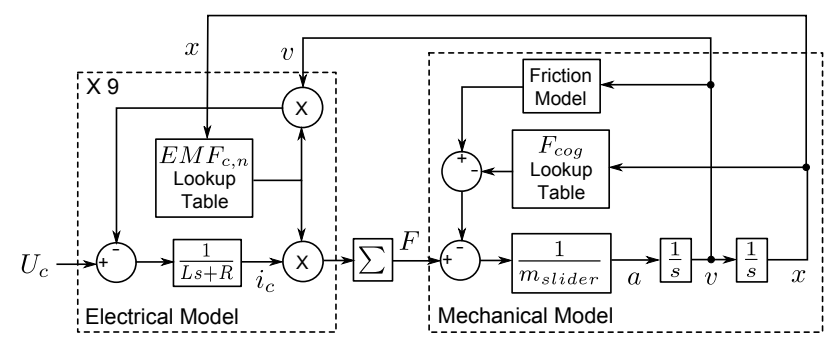

Fig. 10. Electromechanical model of the linear motor.

out of an electrical and mechanical part. The electrical part calculates the force contribution of each coil, supplied with a voltage $U_{c}$, to the total slider force $F$. Parameters include the coil inductance $L=26 \mathrm{mH}$ and resistance $R=1.2 \Omega$. The mechanical model calculates acceleration, speed and position of the slider with mass $m_{\text {slider }}=0.36 \mathrm{~kg}$. It includes coulomb friction $(2 \mathrm{~N})$, damping $(1 \mathrm{~N} / \mathrm{m} / \mathrm{s})$ and cogging force from a $2 \mathrm{D}$ numerical calculation stored in a lookup table. A similar model was created for the three-phase control strategy, taking into account the different coil arrangement (with star point).

\section{B. Simulation Results}

Fig. 11 shows a simulation result, comparing the behaviour of the new multiphase control (left figure) with the conventional three-phase control (right). The linear motor tracks a sawtooth trajectory at $10 \mathrm{~Hz}$ (100 ms back and forth) with a stroke length of $150 \mathrm{~mm}$, i.e. $60 \%$ of maximum stroke $(250 \mathrm{~mm})$. It can be seen that dynamic performance (tracking behaviour) for both control strategies is equal with accelerations up to $100 \mathrm{~g}$. However, a large difference is visible in coil currents. The multiphase control distributes currents optimally over the coils according to (2). Hence, only coils with a useful contribution to the slider force are activated with current proportional to the EMF. Coils which cannot contribute to the force generation (because EMF is nearly zero), conduct no current. Notice that currents in coil 1 and 9 remain zero because the slider never comes at these positions. Three-phase control implies a series connection of coils in three groups (1-4-7, 2-5-8, 3-6-9). Hence, coils not contributing to force generation also conduct current, leading to additional losses.
Fig. 12 shows the loss components for different stroke lengths (per unit) with the conventional three-phase control and the new multiphase control when tracking a $10 \mathrm{~Hz}$ sawtooth profile. Because absolute loss calculations require an extensive model calibration, losses are given in per unit, with base value the loss at a stroke length of 0.6 p.u. This enables an analysis of the influence of both control strategies on losses, without considering absolute values.

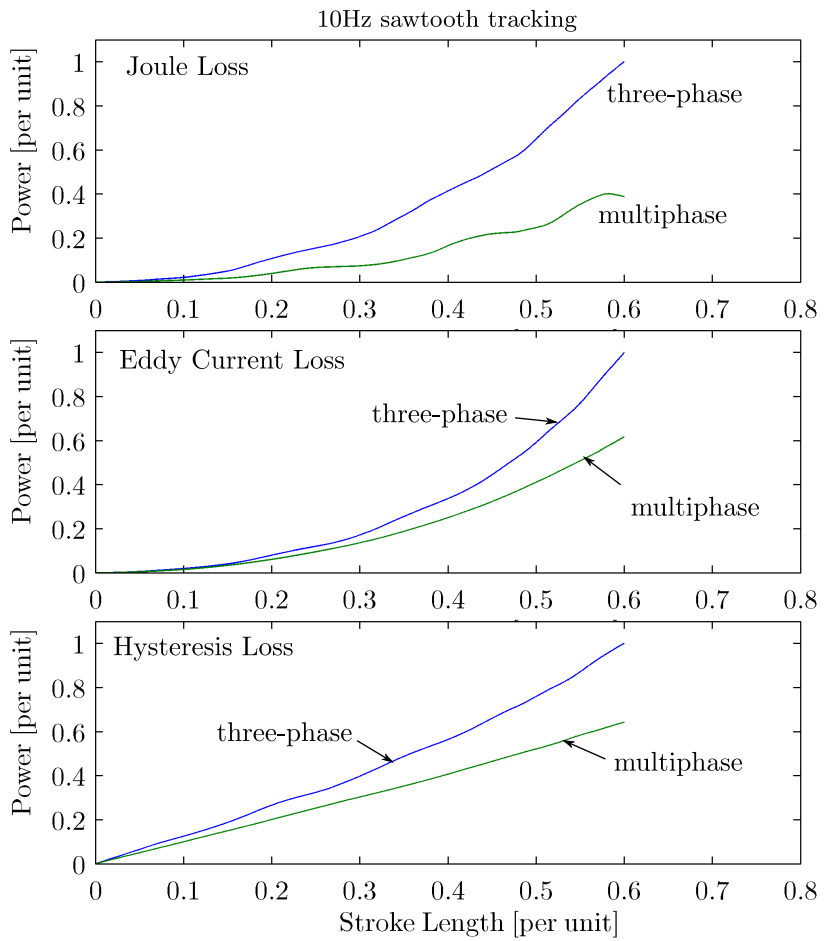

Fig. 12. Simulated per-unit joule, eddy current and hysteresis losses as a function of per-unit stroke length at a $10 \mathrm{~Hz}$ translation frequency (sawtooth position tracking) with three-phase and multiphase control.

The joule losses in the copper coil windings increase quadratically with the stroke length as the required current (acceleration force) scales linearly with stroke length. Notice that at large stroke lengths the required acceleration force would be very large. Excessive joule loss is avoided by the $10 \mathrm{~A}$ current 

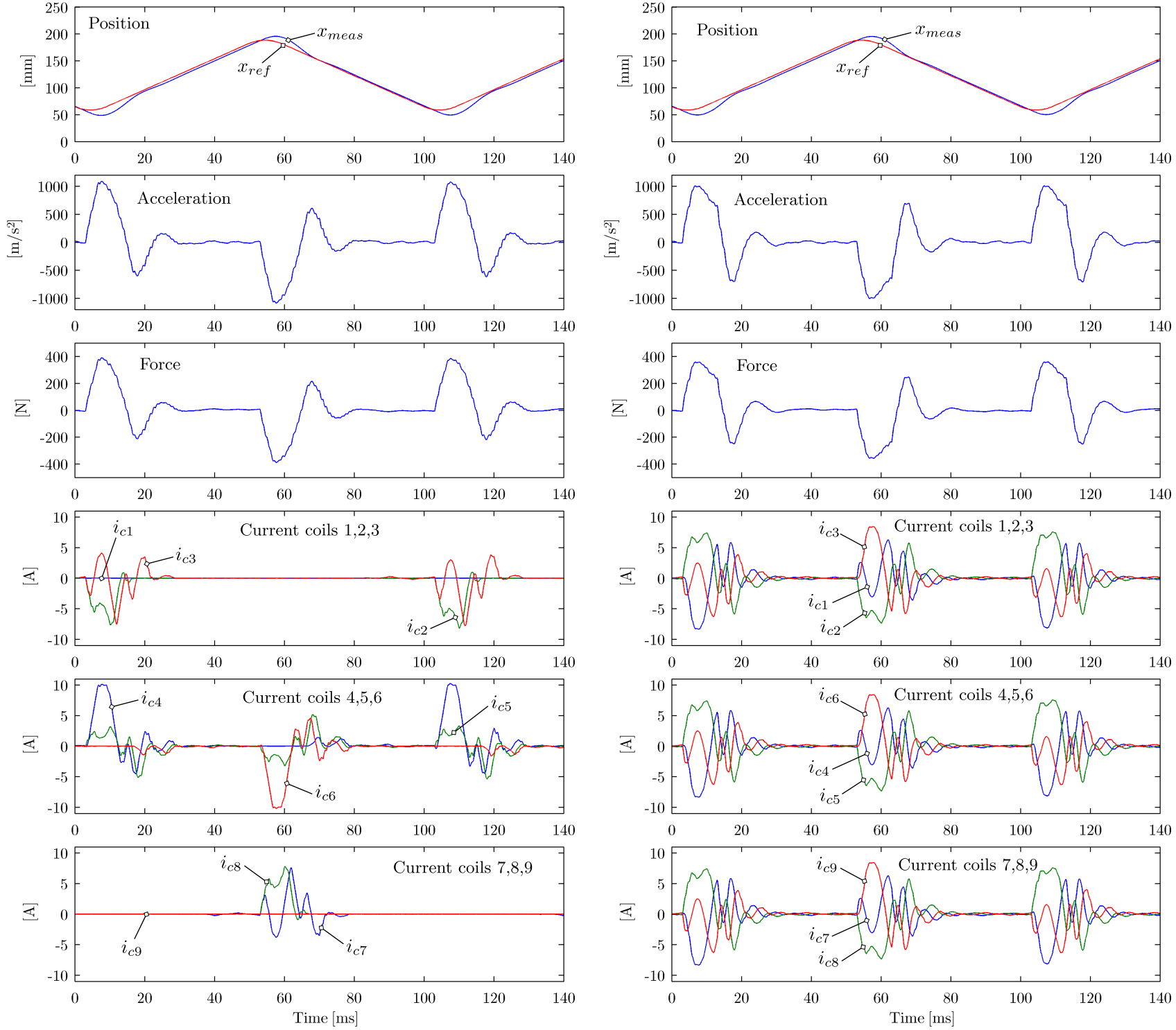
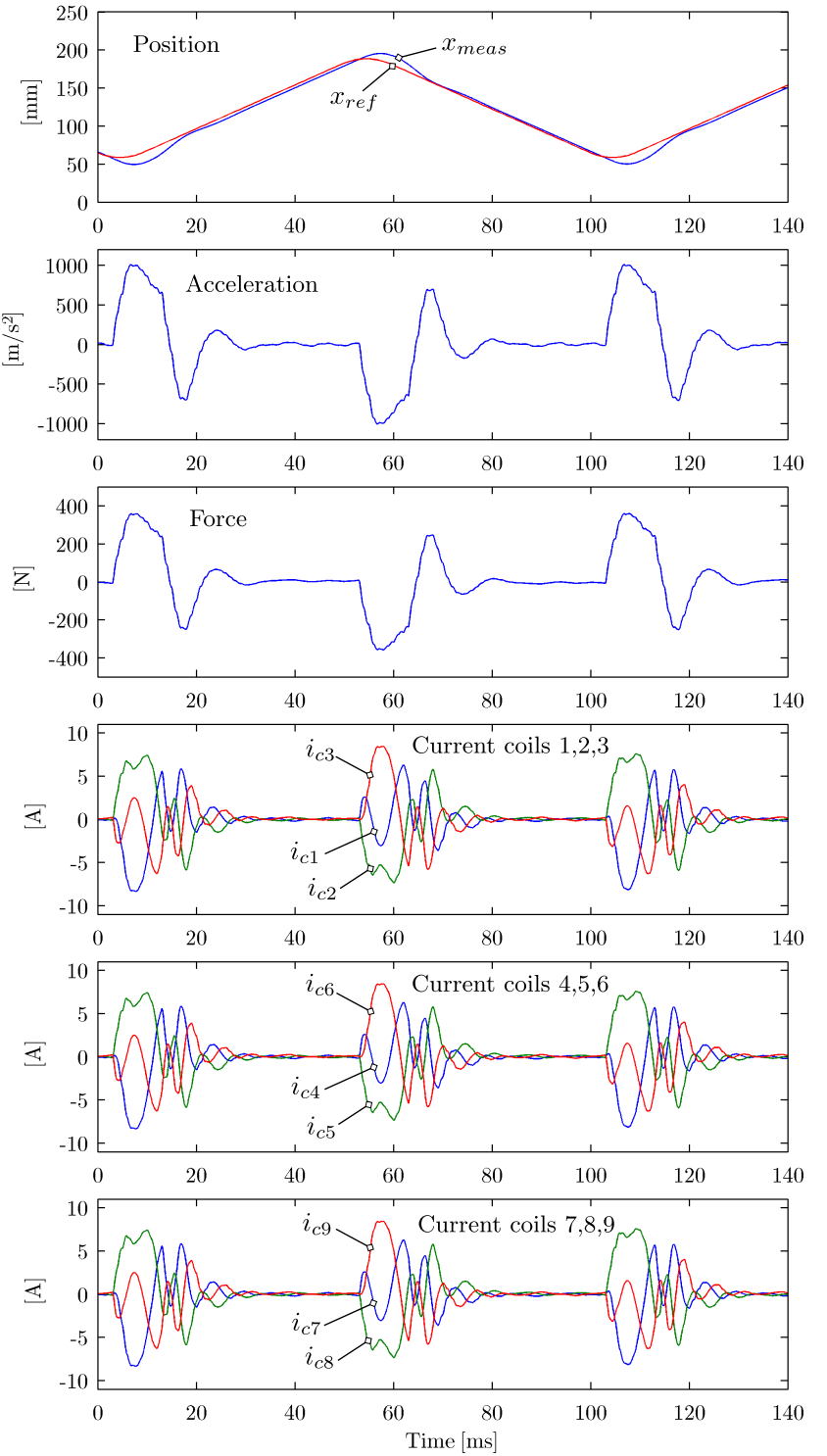

Fig. 11. Simulation of the linear motor tracking a $10 \mathrm{~Hz}$ sawtooth position profile, covering $60 \%$ of total stroke length with multiphase (left) and three-phase (right) control. The coil currents indicate that multiphase control is more efficient for equal dynamic performance.

limit. Comparing both control strategies, the new controller can reduce the joule losses with a factor two thanks to the optimal current distribution strategy. The iron losses (eddy current and hysteresis) were calculated with a coupled SimulinkFEMM numerical simulation. The multiphase control strategy only injects currents in the useful stator coils above the slider so iron losses in other parts of the core material are avoided. Hysteresis losses increase linearly with the stroke length since a larger amount of core material undergoes polarity reversal. Eddy current losses are proportional to the square of change of the magnetic field $(d B / d t)^{2}$. Slider speed has a higher amplitude at larger stroke lengths, hence the electric frequency increases linearly. Hence, eddy current losses will increase quadratically with stroke length.

\section{EXPERIMENTAL RESULTS}

Fig. 14 shows the prototype BLDC linear motor which is driven by a custom-built multiphase converter as shown in Fig. 13. The multiphase control strategy is implemented on a dSPACE rapid prototyping platform, generating PWM signals to control the $\mathrm{H}$-bridges in each phase of the converter. The switching frequency is $6 \mathrm{kHz}$ and the DC supply voltage to each H-bridge is $75 \mathrm{~V}$. To compare the power consumption of the motor with a multiphase controller and a three-phase offthe-shelf drive, the following test was executed. The motor was set to track a sawtooth profile at $10 \mathrm{~Hz}$ for a number of stroke lengths. For each stroke length setting, the drive input power, averaged over 1 second was measured at the DC supply of both converters by means of a Voltech PM100 


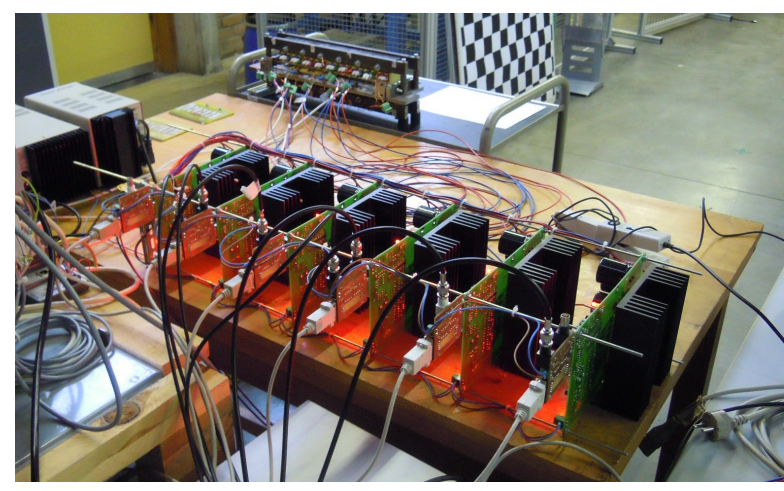

Fig. 13. Custom-built multiphase converter.

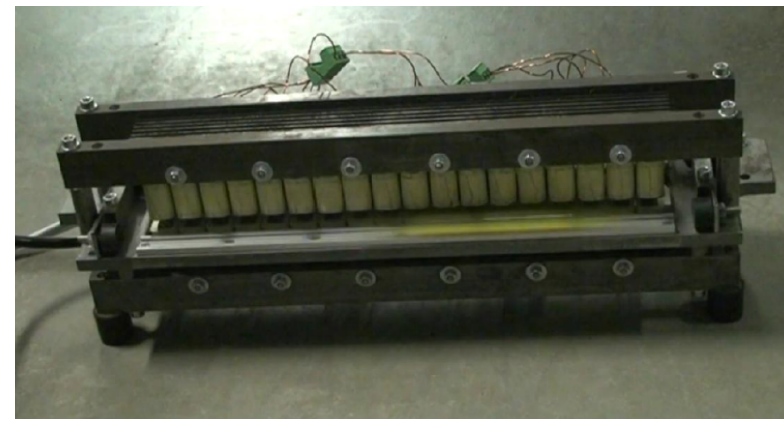

Fig. 14. High-dynamic BLDC linear motor.

power analyzer. At the same time, one second averaged input power to the motor was measured as well. For the threephase drive this is done with a Voltech PM6000 and for the multiphase drive, a 6-channel Yokogawa WT1806. Note that the number of measurement channels (6) restricts the maximum stroke length. Therefore, only 6 out of 9 phases are used. Fig. 15 shows the measured average power values. Note that instantaneous motor input power is pulsating between positive and negative values (accelerating and decelerating). Hence, averaged motor input power entirely consists of iron, joule (largest) and friction losses. Comparing Fig. 15, with the simulation in Fig. 12 reveals the same trend; motor losses (mainly joule) are reduced by a factor two thanks to the optimal current distribution between coils. Converter losses, given by the difference between drive input and motor input power, are in the same order of magnitude.

\section{Conclusion}

Analysis of magnetic field waveforms indicated the inefficiency of three-phase control for the 7-slot 6-pole BLDC linear motor topology. Therefore, an alternative stator coil arrangement and multiphase control strategy is elaborated. Instead of grouping the nine stator coils into three phases, each coil is supplied individually. This allows a more efficient force generation because currents can be distributed optimally between the phases. As the injected current in each coil is made proportional to the normalized electromotive force (at unity velocity), only the useful coils are excitated. Further-

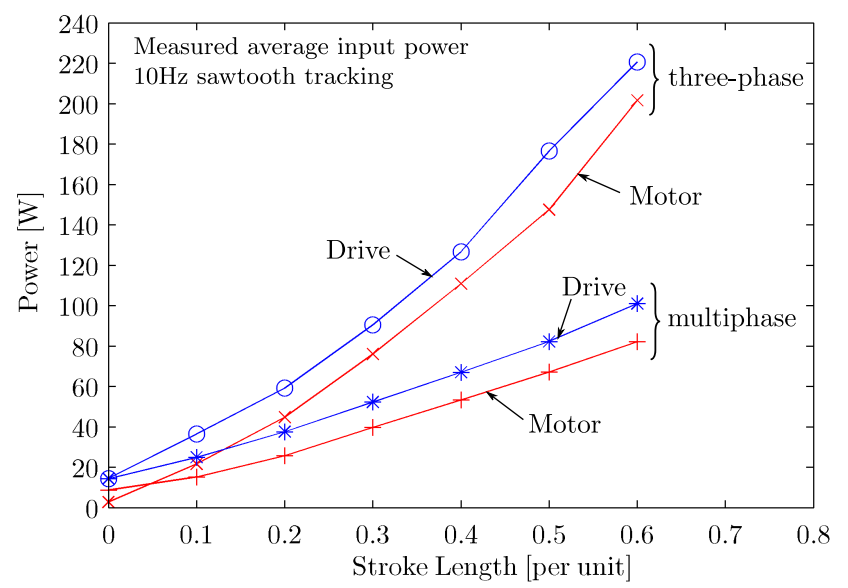

Fig. 15. Measured average motor and drive input power for both three-phase and multiphase control at different stroke lengths. The position profile is a sawtooth with a translation frequency of $10 \mathrm{~Hz}$.

more, coils with the largest potential for force generation at the actual slider position, i.e. with the largest normalized EMF, are supplied with the largest current. The controller includes a lookup table giving the normalized EMF in each coil for a large number of slider positions, as calculated by a numerical 2D simulation. Compared to a conventional threephase control, simulations show a significant reduction of joule and iron losses with multiphase compared to conventional three-phase control. The proposed strategy was implemented on a dSpace rapid prototyping platform, controlling a custombuilt multiphase converter. Input power measurements revealed a $50 \%$ loss reduction with the new controller instead of an offthe-shelf three-phase drive.

\section{REFERENCES}

[1] S. Goossens, W. Verdonck, and W. Symens, "An integrated linear electromagnetic actuator for large motions and extreme accelerations," in The 13th Mechatronics Forum International Conference (Mechatronics 2012), Linz, Austria, sept. 2012.

[2] B. Perreault, "Optimizing operation of segmented stator linear synchronous motors," Proceedings of the IEEE, vol. 97, no. 11, pp. 1777 1785, nov. 2009.

[3] M. Mihalachi, R. Leidhold, and P. Mutschler, "Motion control for long primary linear drives used in material handling," in Power Electronics and Motion Control Conference (EPE/PEMC), sept. 2010, pp. 94-101.

[4] R. Leidhold and P. Mutschler, "Speed sensorless control of a longstator linear synchronous motor arranged in multiple segments," Industrial Electronics, IEEE Transactions on, vol. 54, no. 6, pp. 3246-3254, dec. 2007.

[5] K. Suzuki, Y.-J. Kim, and H. Dohmeki, "Driving method of permanentmagnet linear synchronous motor with the stationary discontinuous armature for long-distance transportation system," Industrial Electronics, IEEE Transactions on, vol. 59, no. 5, pp. 2227-2235, may 2012.

[6] M. Carpita, T. Beltrami, C. Besson, and S. Gavin, "Multiphase active way linear motor: Proof-of-concept prototype," Industrial Electronics, IEEE Transactions on, vol. 59, no. 5, pp. 2178-2188, may 2012.

[7] T. Beltrami, M. Carpita, S. Gavin, and D. Moser, "An active way linear synchronous motor with multiphase independent supply," in International Conference on Electrical Machines (ICEM), sept. 2010, pp. 1-6.

[8] K. Vanvlasselaer and K. Mulier, "Intelligente vermogenselektronica voor rendementsverbetering van een hoogdynamische lineaire motor," Master's thesis, KU Leuven Faculty of Engineering, Heverlee, Belgium, 2012. 